\title{
Possible Linkage Between Osteoporosis and Genes Associated With Type 1 Diabetes Mellitus
}

\author{
Yong $\mathrm{Li}^{1}$, Chengjun $\mathrm{He}^{2}$, Jinhua Sheng ${ }^{3}$, Paul Jones ${ }^{4 *}$ \\ ${ }^{1}$ Department of Orthopedics, The 2nd People's Hospital of Anhui Province, \\ Hefei, Anhui 230000, P.R. China; \\ ${ }^{2}$ Department of Obstetrics and Gynecology, Tongling People's Hospital, Tongli \\ ng, Anhui 244000, P.R. China; \\ ${ }^{3}$ College of Computer Science, Hangzhou Dianzi University, Hangzhou, \\ Zhejiang 310018, P.R. China; \\ ${ }^{4}$ Department of Biology Products, American Informatics Consultant LLC, \\ Rockville, MD, 20852, USA.
}

*Corresponding to: Dr. Paul Jones, Department of Biology Products, American Informatics Consultant LLC, Rockville, MD 20852, USA. Email:p.jones@gousinfo.com; Tel: 1(301)760-3429.

\section{ABSTRACT}

Background: Previous studies suggested that Type 1 diabetes mellitus (T1DM) is related to an increased risk of osteoporosis (OP) with unknown mechanism. Here, we hypothesized that TIDM and OP present shared molecular basis, and that OP related genes may also play roles for TIDM.

Methods: To identify potential OP risk genes from T1DM-gene group, we conducted an integrated analysis using large scale ResNet relation data and gene expression data for T1DM and OP. Disease-gene relation data were acquired from Pathway Studio ResNet Mammalian database. Gene expression profile were acquired from samples of 40 subjects including $20 \mathrm{OP}$ cases and 20 normal controls.

http://mo.qingres.com

\section{GOPEN ACCESS}

DOI: $10.20900 / \mathrm{mo} .20170001$

Received: October 26, 2016

Accepted: December 20, 2016

Published: January 25, 2017

Copyright: (2017 Cain et al. This is an open access article distributed under the terms of the Creative Commons Attribution License, which permits unrestricted use, distribution, and reproduction in any medium, provided the original author and source are credited.
Results: Genes linked to T1DM and OP present significant overlap (183 genes, $p$-value $=2.84 \mathrm{E}-128)$. These genes play roles through multiple genetic pathways (enrichment $p$-value $<8.8 \mathrm{e}-23$ for the top 10 pathways) influencing the pathogenesis of both diseases. A genetic network of 16 genes were identified. T1DM may exert influence on OP these genes. Two T1DM genes, ITGAE and RELB, present significant difference ( $p$-value $<0.0005)$ between OP cases and controls. Network analysis support the results and reveal strong functional association between OP and these two genes.

Conclusion: Results from this study support the hypothesis that complex genetic associations exist between T1DM and OP, and that T1DM related genes should be tested for their potential roles in OP pathogenesis. 
Key words: Type 1 diabetes; Osteoporosisl; ResNet Database; pathway enrichment analysis; Network connectivity analysis

\section{INTRODUCTION}

Type1 diabetes (T1DM) is one type of diabetes mellitus. It is a genetic disease diagnosed in children and young adults characterized by presenting not enough insulin in the body. T1DM accounts for approximately $5 \%$ of all cases of diabetes in USA ${ }^{[1]}$. Studies showed that, T1DM is more frequently linked to fracture risk that that of type 2 diabetes mellitus (T2DM). Studies showed that T1DM is associated with a 6.4 to 6.9 fold increase in the relative risk of hip fracture compared to individuals without diabetes [2].

Osteoporosis (OP) is an age related disorder characterized by low bone mass and increased risk of bone fractures, affecting over 200 million people worldwide ${ }^{[3]}$. People usually reach their peak bone mass by age 30. Approximately $30 \%$ of all postmenopausal women are affected by OP, and $40 \%$ of the population are susceptive to a lifetime risk of a bone fracture ${ }^{[4]}$. The well accepted pathophysiological mechanisms for OP include early apoptosis of osteoblasts ${ }^{[5]}$, osteocytes ${ }^{[6,7]}$, prolongation of the life span of osteoclasts ${ }^{[8]}$ and the imbalance between osteoblastogenesis and adipogenesis of bone marrow mesenchymal stem cells. The functional effect of genes associated with T1DM should be investigated to determine its linkage with OP. Recent genetic studies using both Genomewide association study (GWAS) and gene expression data showed that OP is linked to hundreds of genes ${ }^{[9-11]}$. Additionally, studies showed that TIDM patients tend to demonstrate increased OP risk ${ }^{[12]}$.

In recent years, Pathway Studio ResNet database has been widely used to study modeled relationships between proteins, genes, complexes, cells, tissues and disease ${ }^{[13]}$. In this study, we hypothesized that genetic associations exist between T1DM and OP, and that some genes play roles in the development of TIDM may also influence OP pathogenesis. To test the hypothesis, large scale ResNet relation data and gene expression data were integrated to study the shared genetic basis between TIDM and OP, with the purpose to identify potential OP risk genes.

\section{MATERIALS AND METHODS}

Large scale T1DM-gene and OP-gene ResNet relation data were first studied to identify shared genes and genetic pathways. Then, these OP/ TIDM related genes were further investigated using an OP expression data to discover potential novel genes for OP. After that, functional network analysis were employed to study the potential pathogenic significance of these candidate genes to OP.

\subsection{T1DM-Gene and OP-Gene data acquisition}

Disease-gene relation data for both T1DM and OP were acquired from Pathway Studio (PS) ResNet relation database. Updated weekly, The PS ResNet Databases is the largest database among known competitors in the field ${ }^{[14]}$. Besides the full lists of genes, we also presented the supporting references for each disease-gene relation in Supplementary Data, including titles of the references and the related sentences where these relations were identified. These information could be used to locate detailed description of how a candidate gene is related to T1DM and/or OP.

\subsection{Identification of OP risk genes}

We used a gene expression data set (GSE13850) of 40 subjects to test the genes related to T1DM but not to OP. The gene expression profile were acquired from 20 smoking-related OP in postmenopausal females and 20 healthy postmenopausal females (GSE7429). The expression data were log2 normalized and is online available at https://www. ncbi.nlm.nih.gov/geo/query/acc.cgi?acc=GSE13850. For each gene that linked to TIDM but not to OP, a one-way ANOVA analysis were performed on OP cases and controls. FDR corrected $p$-values were used to pick potential OP risk genes for further analysis.

\subsection{Network analysis of OP risk genes}

For the target OP risk genes identified through expression analysis described above, we performed a network analysis between the target genes and OP to identify potential biological connections. The analysis were performed using Pathway Studio (www.pathwaystudio.com) at three levels: proteins/ genes, small molecular/drugs and functional classes. 


\section{and OP}

A systematic analysis on the T1DM-Gene and OPGene ResNet relation data was conducted. Results showed that 977 genes were associated with T1DM, supported by 4,454 references from 1975 to Oct. 2016 (Supplementary Table S1a and Table S1b). For OP, we identified 507 genes supported by 2,321 references from 1976 to Oct. 2016 (Supplementary Table S2a and Table S2b). A significant overlap of 183 genes appears between the T1DM-genes and OP-genes $(p$-value $=2.84 \mathrm{E}-128 ;$ Right tail Fisher's Exact test,), as shown in Fig. 1. The shared genes and related references appear in Supplementary Table S3a and Table S3b.

To test the functional profile of the 183 genes associated with both T1DM and OP, we conducted a Pathway Enrichment Analysis (PEA) using Pathway Studio. The 10 most significantly enriched pathways $(p$-value $<8.8 \mathrm{e}-23)$ are presented in Table 1. In

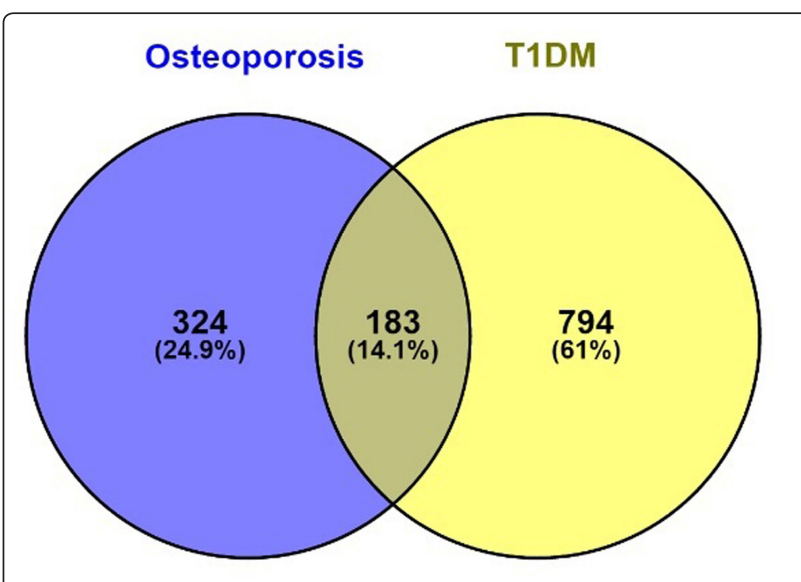

Fig.1 Venn diagram for overlap between T1DMgenes and OP-genes

total, 615 pathways/gene sets were enriched with $p$-value $<1 \mathrm{e}-04$ including 179 out of these 183 genes (Supplementary Table S4).

Table 1. Genetic pathways enriched with 183 genes linked to both T1DM and OP

\begin{tabular}{|c|c|c|c|c|c|}
\hline Name & GO ID & \# of Entities & Overlap & $\begin{array}{c}p \text {-value } \\
\text { (FDR) }\end{array}$ & $p$-value 0 \\
\hline aging & 0016280 & 254 & 46 & $1.72 \mathrm{E}-43$ & $5.82 \mathrm{E}-48$ \\
\hline response to hypoxia & 0001666 & 259 & 41 & $5.82 E-36$ & $3.93 \mathrm{E}-40$ \\
\hline response to lipopolysaccharide & 0032496 & 252 & 38 & 2.43E-32 & $2.46 \mathrm{E}-36$ \\
\hline inflammatory response & 0006954 & 404 & 43 & $1.08 \mathrm{E}-30$ & $1.45 \mathrm{E}-34$ \\
\hline response to drug & 0017035 & 509 & 46 & 4.87E-30 & 8.23E-34 \\
\hline $\begin{array}{l}\text { positive regulation of cell } \\
\text { proliferation }\end{array}$ & 0008284 & 568 & 46 & 5.39E-28 & 1.09E-31 \\
\hline cell surface & 0009929 & 645 & 43 & $3.00 \mathrm{E}-23$ & $7.80 \mathrm{E}-27$ \\
\hline $\begin{array}{l}\text { response to organic cyclic } \\
\text { compound }\end{array}$ & 0014070 & 253 & 31 & $3.00 \mathrm{E}-23$ & 8.11E-27 \\
\hline response to glucocorticoid & 0051384 & 133 & 25 & 4.84E-23 & 1.47E-26 \\
\hline response to estrogen & 0043627 & 104 & 23 & $8.85 \mathrm{E}-23$ & $2.99 \mathrm{E}-26$ \\
\hline
\end{tabular}

Note: For each pathway/Go term, the $p$-value was calculated using Fisher-Exact test against the hypothesis that a randomly selected gene group of same size (183) can generate a same or higher overlap with the corresponding pathway/Go term. All these pathways/Go terms passed the FDR correction ( $q=0.001)$. 
Many of these pathways have been implicated with both T1DM and OP, such as the response to lipopolysaccharide (GO ID: 0032496) ${ }^{[15,16]}$, aging (GO ID: 0016280 ${ }^{[17,18]}$, response to estrogen (GO ID: 0043627) ${ }^{[19,20]}$, and inflammatory response (GO ID: 0006954) ${ }^{[21,22]}$. For more detailed information of these significantly enriched pathways, please refer to Supplementary Table S4. Our results suggest that T1DM and OP share multiple genetic pathways, through which a large group of genes play roles affecting the pathogenic development of both diseases.

\subsection{Possible T1DM $\rightarrow$ OP regulations}

Further functional network analysis using PS showed that, 16 out of the 183 genes are downstream targets of T1DM (influenced by T1DM), while they are the upstream regulator of OP, as shown in Fig. 2. Therefore, T1DM may influence the pathogenic development of OP through the regulation of these 16. For each relation (arrow) in Fig. 2, there were one or more supporting references (see Supplementary Table S3b), which could be used for detailed description of each relation.
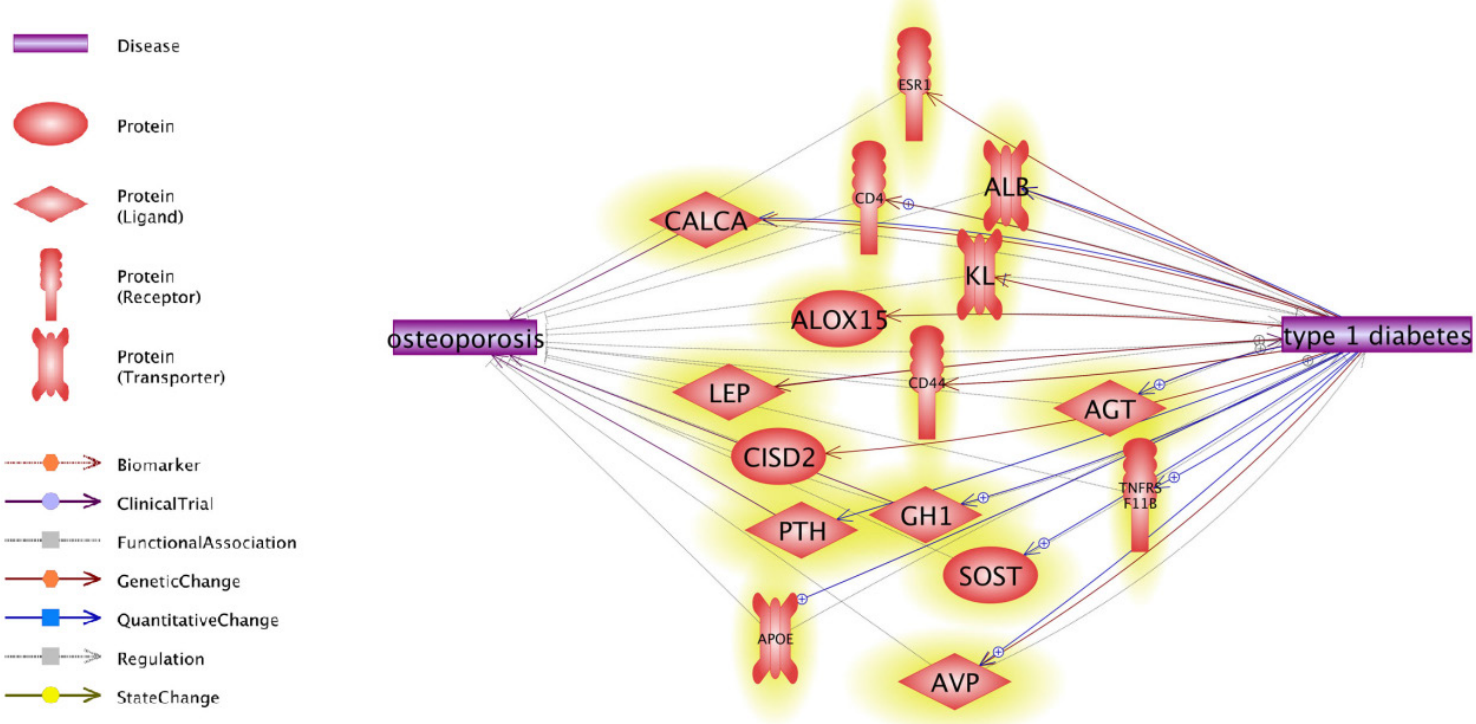

Fig. 2 A T1DM $\rightarrow$ Gene $\rightarrow$ OP pathway composed of 16 genes. The network were generated using 'network building' module of Pathway Studio. For the definition of the entity types and relation types in the figure please refer to http://pathwaystudio.gousinfo.com/ResNetDatabase.html

Our results suggest that any genes linked to T1DM may be worthy of study for their potential relation to OP. These genes affect the pathogenic development of T1DM, which in turn may influence the disease status of OP.

\subsection{Gene expression analysis of T1DM- genes for OP}

Fig. 1 showed that more T1DM genes were not linked to OP than these were (794 vs. 183). To identify genes only linked to T1DM and may be potentially OP risk genes, a gene expression analysis was conducted to study the expression difference between OP case and controls (NCBI GSE13850) on these 794 genes (see Supplementary Table S5 for results). Fig. 3 elucidates the normalized '-log10' transferred $p$-values (NLP) of each gene, where the transferred $p$-value was normalized by the mean $p$-value of all genes, such that genes with mean '-log10' transferred $p$-values will have a NLP $=1$.

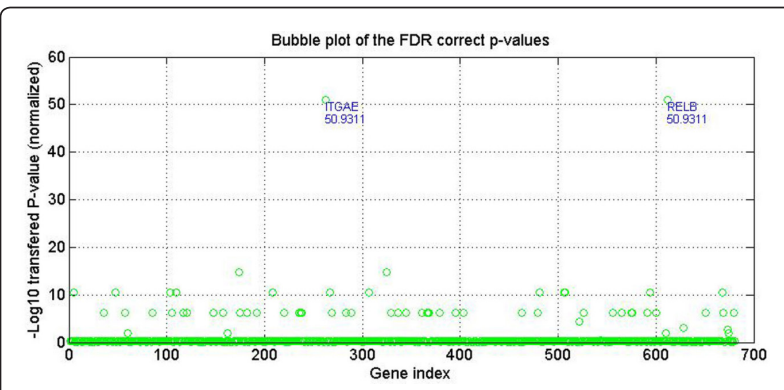

Fig. 3 The $p$-values of the 794 T1DM genes for OP case/control expression comparison. The $p$-values have been through FDR correction with $q=0.05$ and normalized logic transformation using '-log10'. Names of 2 genes demonstrating significant differences ( $p$-value $<0.0005)$ were marked at corresponding positions. 
The two genes, ITGAE and RELB, presented significant difference ( $p$-value $<0.0005$, before FDR correction) between OP cases and controls. According to PS ResNet database (updated Nov. 2016), these two genes present no direct relation with OP (no reference reporting an association between these genes and OP). However, further network analysis revealed strong functional association between these two genes and OP, bridged by 36 genes/proteins and 3 small molecular (see Fig. 4). The 41 entities and the 82 relations with 474 supporting references in Fig. 4 are presented in Supplementary Table S6a and S6b, respectively.

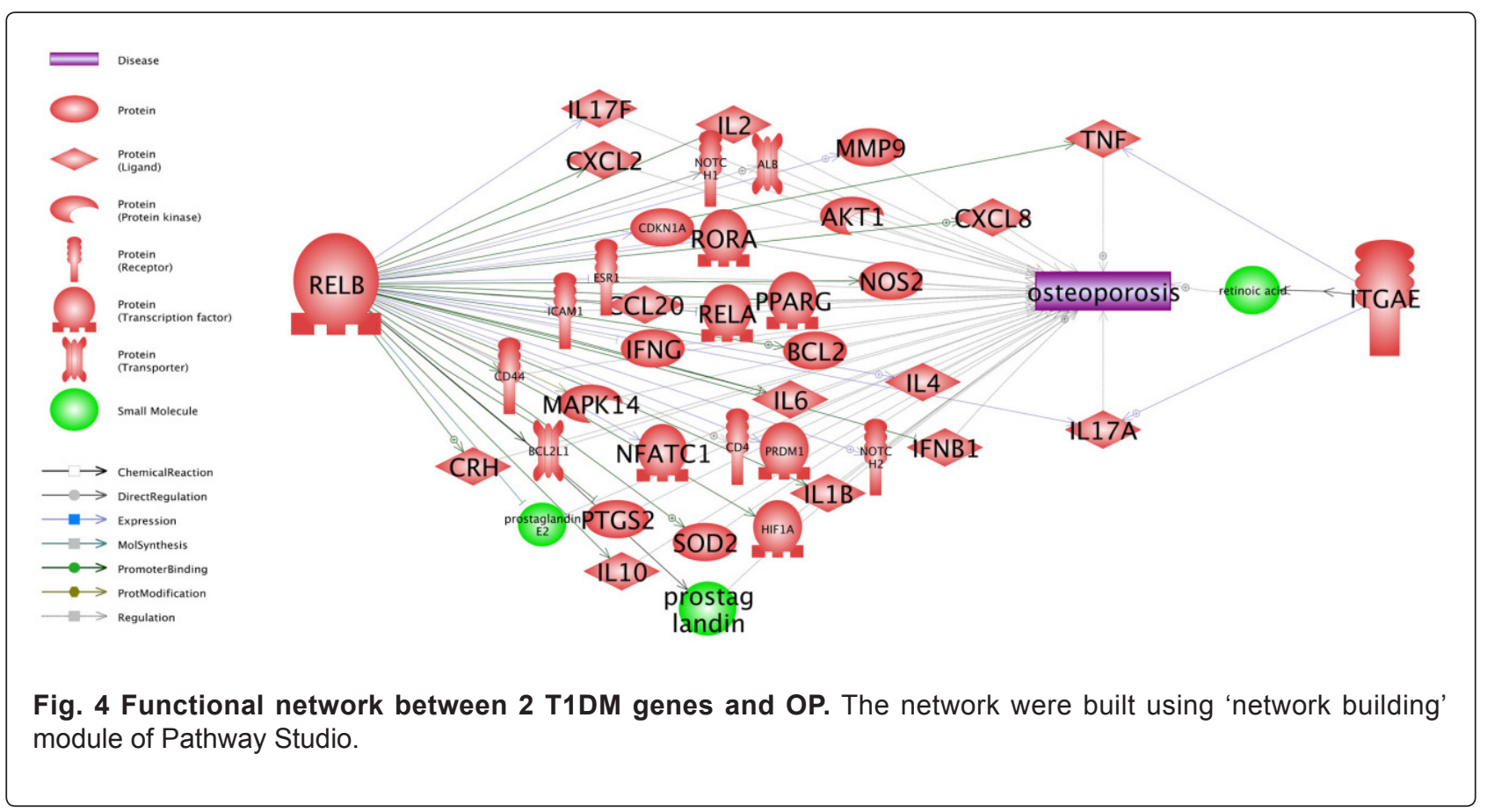

\section{DISCUSSION}

Previous studies showed that T1DM is strongly linked to increase incidence of OP with unclear mechanism. In this study, we used large scale ResNet relation data and gene expression data to study the share genes and genetic pathway between T1DM and OP, based on which we identified potential novel risk genes for OP.

Our results showed that genes linked to T1DM and OP present significant overlap (183 genes, $p$-value $=2.84 \mathrm{e}-128$ ). Furthermore, 179 out of these 183 genes were significantly enriched within 615 pathways ( $p$-value < 1e-04, FDR corrected: $q=$ $0.005)$, many of which have been implicated to be linked to both T1DM and OP, such as the response to lipopolysaccharide (GO ID: 0032496) ${ }^{[15,16]}$, aging (GO ID: 0016280) ${ }^{[17,18]}$, response to estrogen (GO ID: 0043627) ${ }^{[19,20]}$, and inflammatory response (GO ID: 0006954) ${ }^{[21,22]}$. These results suggest that T1DM and OP share multiple genetic pathways. A large group of genes (179 out of 183) regulate the pathogenic development of both diseases through these pathways. Moreover, we observed a 16gene network, through which T1DM could affect the disease status of OP (Fig. 2). Our findings provide further support for the hypothesis that T1DM genes may also regulate pathogenic development of OP.

Closer study of the 794 T1DM alone genes (Fig. 1 (a)) using OP gene expression data showed that two genes, ITGAE and RELB, demonstrated significant difference between OP cases and controls ( $p$-value $<0.0005$, NLP $=50.93$; in Fig. 3. Further validation using ResNet network analysis showed that, these two genes presented strong functional correlation with OP, forming a functional genetic network supported by 474 supporting references (Fig. 4 and Supplementary Tables S6b). Specially, RELB presented strong correlation with OP through 38 genes/proteins and 2 small molecules. Where there are there two genes and one molecule linking it to OP. The network analysis revealed multiple potential pathways through which ITGAE and RELB may affect the disease status of OP. In additionally, the RELB is essential for the regulation of IL17A production in GD T cells ${ }^{[23]}$, while IL17A mediated SRANK ligand elevation is involved in postmenopausal $\mathrm{OP}^{[24]}$. These findings indicates a 
possible RELB $\rightarrow$ IL 17A $\rightarrow$ OP regulatory pathway. A possible ITGAE $\rightarrow$ TNF $\rightarrow$ OP regulatory pathway was identified as follows. ITGAE coded integrin protein CD103 (cluster of differentiation 103) expresses in many dendritic cells (e.g., CD103-DCs), influencing the production of tumor necrosis factor alpha (TNF- $\alpha$ ) ${ }^{[25]}$, which plays a pivotal role in inflammationrelated OP through the promotion of bone resorption and suppression of bone formation ${ }^{[26]}$. These observations suggest a possible TNF- $\alpha$ pathway via which ITGAE may regulate OP. More potential connections between these two genes and OP could be identified in Supplementary Tables S6b.

To sum up, results from this study support the hypothesis that T1DM and OP present significant associations at genetic level, which may partially explain their clinical correlations. Moreover, novel potential OP genes can be identified by cross disease analysis integrating ResNet relation data and gene expression data. To our knowledge, this is the first study integrating large scale ResNet relation data and gene expression data to study the molecular associations between T1DM and OP. Findings here may provide new insights into the current field of T1DM-OP correlation study, and guarantee further studies using more data sets to identify novel potential risk genes for OP.

\section{ACKNOWLEDGEMENT}

We would like to thank all authors for their contribution to this study.

\section{CONFLICT OF INTERESTS}

The authors declaim no conflict of interests.

\section{REFERENCES}

1. Centers for Disease Control and Prevention. National diabetes fact sheet: national estimates and general information on diabetes and prediabetes in the United States, 2011. Atlanta, GA: U.S. Department of Health and Human Services, Centers for Disease Control and Prevention; 2011. Available from: http://www. cdc.gov/diabetes/pubs/pdf/ndfs_2011.pdf.

2. Vestergaard P. Discrepancies in bone mineral density and fracture risk in patients with type 1 and type 2 diabetes \& a meta-analysis. Osteoporos Int. 2007; 18(4): 427-444.

3. Reginster JY, Burlet N. Osteoporosis: a still increasing prevalence. Bone. 2006; 38(2 Suppl 1): S4-9.
4. Melton LJ 3rd, Chrischilles EA, Cooper C, Lane AW, Riggs BL. Perspective. How many women have osteoporosis? J Bone Miner Res. 1992; 7(9): 1005-1010.

5. Manolagas SC, Kousteni S, Jilka RL. Sex steroids and bone. Recent Prog Horm Res. 2002; 57: 385-409.

6. Tomkinson A, Gevers EF, Wit JM, Reeve J, Noble BS. The role of estrogen in the control of rat osteocyte apoptosis. J Bone Miner Res.1998; 13: $1243-1250$.

7. Tomkinson A, Reeve J, Shaw RW, Noble BS. The death of osteocytes via apoptosis accompanies estrogen withdrawal in human bone. J Clin Endocrinol Metab. 1997; 82: 3128-3135.

8. Boyce BF, Hughes DE, Wright KR, Xing L, Dai A. Recent advances in bone biology provide insight into the pathogenesis of bone diseases. Lab Invest. 1999; 79: 83-94.

9. Richards JB, Zheng HF, Spector TD. Genetics of osteoporosis from genome-wide association studies: advances and challenges. Nat Rev Genet. 2012; 13(8): 576-588.

10. Mori S. Genome-wide association study for Osteoporosis. Clin Calcium. 2016; 26(4): 537543.

11. Yu G, Wang L, Li Y, Ma Z, Li Y. Identification of drug candidate for osteoporosis by computational bioinformatics analysis of gene expression profile. Eur J Med Res. 2013; 18: 5.

12. Hofbauer LC, Brueck CC, Singh SK, Dobnig H. Osteoporosis in patients with diabetes mellitus. $\mathrm{J}$ Bone Miner Res. 2007; 22: 1317-1328.

13. Nikitin A, Egorov S, Daraselia N, Mazo I. Pathway studio-the analysis and navigation of molecular networks. Bioinformatics. 2003; 19(16): 2155-2157.

14. Lorenzi PL, Claerhout $S$, Mills GB, Weinstein $\mathrm{JN}$. A curated census of autophagy-modulating proteins and small molecules: candidate targets for cancer therapy. Autophagy. 2014; 10(7): 1316-1326.

15. Nakata $\mathrm{K}$, Nakata $\mathrm{Y}$, Inagawa $\mathrm{H}$, Nakamoto $\mathrm{T}$, Yoshimura H, Soma G. Pantoea agglomerans lipopolysaccharide maintains bone density in premenopausal women: a randomized, doubleblind, placebo-controlled trial. Food Sci Nutr. 2014; 2(6): 638-646.

16. Beyan H, Goodier MR, Nawroly NS, Hawa MI, Bustin SA, Ogunkolade WB, Londei M, Yousaf $\mathrm{N}$, Leslie RD. Altered monocyte cyclooxygenase response to lipopolysaccharide in type 1 
diabetes. Diabetes. 2006; 55(12): 3439-3445.

17. Veldurthy V, Wei R, Oz L, Dhawan P, Jeon $\mathrm{YH}$, Christakos S. Vitamin D, calcium homeostasis and aging. Bone Res. 2016; 4: 16041.

18. Samos LF, Roos BA. Diabetes mellitus in older persons. Med Clin North Am. 1998; 82: 791-803.

19. Codner E. Estrogen and type1 diabetes mellitus. Pediatr Endocrinol Rev. 2008; 6(2): 228-234.

20. Riggs $B L$. The mechanisms of estrogen regulation of bone resorption. J Clin Invest. 2000; 106(10): 1203-1204.

21. Yun AJ, Lee PY. Maladaptation of the link between inflammation and bone turnover may be a key determinant of osteoporosis. Med Hypotheses. 2004; 63: 532-537.

22. Baumann B, Salem HH, Boehm BO. Antiinflammatory therapy in type 1 diabetes. Curr Diab Rep. 2012; 12(5): 499-509.

23. Brüstle A, Brenner D, Knobbe CB, Lang PA, Virtanen C, Hershenfield BM, Reardon C,
Lacher SM, Ruland J, Ohashi PS, Mak TW. The NF-KB regulator MALT1 determines the encephalitogenic potential of Th17 cells. J Clin Invest. 2012; 122(12): 4698-4709.

24. Molnár I, Bohaty I, Somogyiné-Vári É. IL-17Amediated SRANK ligand elevation involved in postmenopausal osteoporosis. Osteoporos Int. 2014; 25(2): 783-786.

25. Coombes JL, Siddiqui KR, Arancibia-Cárcamo CV, Hall J, Sun CM, Belkaid Y, Powrie F. A functionally specialized population of mucosal CD103+ DCs induces Foxp3+ regulatory T cells via a TGF-beta and retinoic acid-dependent mechanism. J Exp Med. 2007; 204(8): 17571764.

26. Lian C, Wu Z, Gao B, Peng Y, Liang A, Xu C, Liu L, Quu X, Huang J, Zhou H, Cai Y, Su P, Huang D. Melatonin reversed tumor necrosis factor-alphainhibited osteogenesis of human mesenchymal stem cells by stabilizing SMAD1 protein. J Pineal Res. 2016; 61(3): 317-327. 